\title{
INEQUALITIES OF APPELL'S HYPERGEOMETRIC FUNCTIONS OF TWO VARIABLES
}

\author{
C. M. JOSHI* AND S. K. BISSU
}

\begin{abstract}
Inequalities for $F_{4}$ for positive and negative real arguments has been obtained using the two sided inequalities of ${ }_{0} F_{1}$ 's which are in the integrand of its Laplace type integral representation. Also incorporated in the discussion are new inequalities for $F_{1}, F_{2}$ and $F_{3}$ which have the advantage over Luke's inequalities in the sense that these hold in a wider domain. Verifications of these bounds have been pointed out numerically and further it is observed that in some cases even under Luke's conditions our results give sharper bounds.
\end{abstract}

\section{Introduction}

It was Luke [10] to use the concept of Pad'e approximations in obtaining inequalities of generalized hypergeometric functions and further to exploit [11] these notions in establishing inequalities of Appell's hypergeometric functions of two variables viz; $F_{1}$, $F_{2}$ and $F_{3}$ through their Eulerian integral representations for negative real arguments. He had to limit his discussion to the inequalities of $F_{1}, F_{2}$ and $F_{3}$, for his effort in obtaining an inequality for $F_{4}$ through Burchnall and Chaundy's ([5], p.261 (68)] integral representation:

$$
\begin{aligned}
& \frac{\Gamma(\alpha) \Gamma(\beta) \Gamma(\gamma-\alpha)(\gamma-\beta)}{\Gamma(\gamma) \Gamma\left(^{\prime}\right)} F_{4}\left[\alpha, \beta ; \gamma, \gamma^{\prime} ; x(1-y), y(1-x)\right] \\
= & \int_{0}^{1} \int_{0}^{1} u^{\alpha-1} v^{\beta-1}(1-v)^{\gamma^{\prime}-\beta-1}(1-u)^{\gamma-\alpha-1}(1-u x)^{\alpha-\gamma-\gamma^{\prime}+1}(1-v y)^{\beta-\gamma-\gamma^{\prime}+1} \\
& \quad \times(1-u x-v y)^{\gamma+\gamma^{\prime}-\alpha-\beta-1} d u d v \\
& \operatorname{Re}(\gamma)>\operatorname{Re}(\alpha)>0, \operatorname{Re}\left(\gamma^{\prime}\right)>\operatorname{Re}(\beta)>0,|x|<\rho,|y|<\rho, \rho+\rho^{\prime}<1 \\
& \sqrt{\left\{\rho\left(1+\rho^{\prime}\right)\right\}}+\sqrt{\left\{\rho^{\prime}(1+\rho)\right\}}<1,
\end{aligned}
$$

which is essentially the same as that of Bailey ([4] p.14(3.2)), could not lead to a simple inequality because of the presence of multiplicity of binomial functions involved in the integrand (1.1). Recently, we [8] have obtained inequalities for Appell's hypergeometric functions $F_{1}, F_{2}, F_{3}$ and $F_{4}$ for positive real arguments.

Received June 26, 1995.

1991 Mathematics Subject Classification. Primary 33C65, Secondary 26D15.

Key words and phrases. .

* This Research is supported by UGC grant No. F:8-13/87(SR-III). 
In this note we overcome this difficulty and obtain the inequalities for $F_{4}$ for positive and negative real arguments using the two sided inequalities of ${ }_{0} F_{1}$ 's which are in the integrand of its integral representations. Also incorporated in the discussion are new inequalities for $F_{1}, F_{2}$ and $F_{3}$ and these inequalities have the advantage over Luke's inequalities in the sense that these hold under weaker conditions and further it is observed that in some cases even under Luke's conditions our results give sharper bounds than those of Luke [11]. The advantage of these inequalities for positive arguments over previous known results is that in the first place they are precise in contrast to unwieldy results available earlier and secondly hold in the extended domain.

In the investigation that follows we shall require:

Lemma 1. If $a>0, z>0$, then

$$
1-\frac{z}{a}<{ }_{0} F_{1}(-; a ;-z)<1-\frac{z}{a}+\frac{z^{2}}{2(a)_{2}} .
$$

Proof. The proof is straight forward and in fact follows from the integral representation ([6], Vol. I p.255(1))

$$
{ }_{1} F_{1}(a ; c ;-z)=\frac{\Gamma(c)}{\Gamma(a) \Gamma(c-a)} \int_{0}^{1} e^{-z t} t^{a-1}(1-t)^{c-a-1} d t, \quad c>a>0,
$$

the inequality

$$
1-z<e^{-z}<1-z+\frac{z^{2}}{2}, \quad z>0
$$

beta integral representation ([6], Vol. I, p.9 (59)) and the confluence principle.

Lemma 2. If $a>0,0<z<1$, then

$$
1+\frac{z}{a}<{ }_{0} F_{1}(-; a ; z)<1+\frac{2 z}{a} .
$$

Proof is on similar lines to that of Lemma 1, but instead of (1.4), we use the inequality

$$
1+z<e^{z}<1+2 z, 0<z<1
$$

which is an out come of Luke's result ([9], p.195 (5)).

\section{Inequalities for $F_{4}$}

The function $F_{4}$ defined by ([6], Vol. I, p.224 (9))

$$
F_{4}=F_{4}\left(\alpha, \beta, ; \gamma, \gamma^{\prime} ;-x,-y\right)=\sum_{m, n=0}^{\infty} \frac{(\alpha)_{m+n}(\beta)_{m+n}}{(\gamma)_{m}\left(\gamma^{\prime}\right)_{n}} \cdot \frac{(-x)^{m}}{m !} \cdot \frac{(-y)^{n}}{n !}
$$


neither $\gamma$ nor $\gamma^{\prime}$ is a negative integer or zero, has the integral representation

$$
F_{4}=\frac{1}{\Gamma(\alpha) \Gamma(\beta)} \int_{0}^{\infty} \int_{0}^{\infty} e^{-(u+v)} u^{\alpha-1} v^{\beta-1}{ }_{0} F_{1}(-; \gamma ;-x u v)_{0} F_{1}\left(-; \gamma^{\prime} ;-y u v\right) d u d v
$$

provided $\alpha>0, \beta>0$, which is based on the observation

$$
(\lambda)_{m}=\frac{1}{\Gamma(\lambda)} \int_{0}^{\infty} e^{-t} t^{\lambda+m-1} d t, R(\lambda)>0, m=0,1,2, \cdots
$$

Appropriate applications of (1.2) and (2.3) in (2.2) would give

Theorem 1. If $\alpha>0, \beta>0, \gamma>0, \gamma^{\prime}>0, x>0, y>0$, then

$$
\begin{aligned}
& 1-\left(\frac{x}{\gamma}+\frac{y}{\gamma^{\prime}}\right) \alpha \beta+\frac{x y(\alpha)_{2}(\beta)_{2}}{\gamma \gamma^{\prime}} \\
< & F_{4}\left(\alpha, \beta ; \gamma, \gamma^{\prime} ;-x,-y\right) \\
< & 1-\left(\frac{x}{\gamma}+\frac{y}{\gamma^{\prime}}\right) \alpha \beta+\frac{x y(\alpha)_{2}(\beta)_{2}}{\gamma \gamma^{\prime}}+\frac{x^{2}(\alpha)_{2}(\beta)_{2}}{2(\gamma)_{2}} \\
& +\frac{y^{2}}{2\left(\gamma^{\prime}\right)_{2}}\left((\alpha)_{2}(\beta)_{2}+\frac{x^{2}(\alpha)_{4}(\beta)_{4}}{2(\gamma)_{2}}\right)-\frac{x y(\alpha)_{3}(\beta)_{3}}{2 \gamma \gamma^{\prime}}\left(\frac{x}{\gamma^{+2}}+\frac{y}{r^{\prime+2}}\right) .
\end{aligned}
$$

Proceeding in a similar fashion, but using (1.5) instead of (1.2) in the integral representation for positive argument, we have

Theorem 2. If $\alpha>0, \beta>0, \gamma>0, \gamma^{\prime}>0,0<x<1,0<y<1, \sqrt{x}+\sqrt{y}<1$, then

$$
\begin{aligned}
1+\alpha \beta\left(\frac{x}{\gamma}+\frac{y}{\gamma^{\prime}}\right)+\frac{x y(\alpha)_{2}(\beta)_{2}}{\gamma \gamma^{\prime}} & <F_{4}\left(\alpha, \beta ; \gamma, \gamma^{\prime} ; x, y\right) \\
& <1+2 \alpha \beta\left(\frac{x}{\gamma}+\frac{y}{\gamma^{\prime}}\right)+\frac{4 x y(\alpha)_{2}(\beta)_{2}}{\gamma \gamma^{\prime}}
\end{aligned}
$$

In particular for the set of values $\alpha=.3, \beta=.5, \gamma=2, \gamma^{\prime}=2.5, x=.2, y=.1$, we have from (2.4)

$$
.98017<F_{4}\left(\alpha, \beta ; \gamma, \gamma^{\prime} ;-x,-y\right)=.98097<.9811062
$$

and from (2.5)

$$
1.02217<F_{4}\left(\alpha, \beta ; \gamma, \gamma^{\prime} ; x, y\right)=1.023874<1.04668
$$

In this context it will be pertinent to remark that Bailey [1-3], Waston [13-14] and Bateman (see for instance [6]) brought out connection of $F_{4}$ with Jacobi polynomials and Bessel functions. One can therefore either use inequalities of ${ }_{2} F_{1}$ 's given by Luke [10] or that of ${ }_{0} F_{1}$ 's obtained above, to get the new inequalities of $F_{4}$ but inequalities obtained will be for a particular form of $F_{4}$. For instance Bailey's result ([1], p.306 (2.1) 
and see also [2]) which expresses $F_{4}$ as product of two ${ }_{2} F_{1}$ 's would give an inequality for $F_{4}$ in the form

$$
\begin{aligned}
\left(1-\theta_{1} x\right)^{-\alpha}\left(1-\theta_{2} y\right)^{-\alpha} & <F_{4}(\alpha, \beta ; \gamma, \alpha+\beta-\gamma+1 ; x(1-y), y(1-x)) \\
& <\left(1-\theta_{1}+\theta_{1}(1-x)^{-\alpha}\right)\left(1-\theta_{2}+\theta_{2}(1-y)^{-\alpha}\right)
\end{aligned}
$$

where

$$
\begin{aligned}
& \theta_{1}=\frac{\beta}{\gamma}, \quad \theta_{2}=\frac{\beta}{\alpha+\beta-\gamma+1}, \quad \alpha>0,0<\beta<\min \{r, \alpha+\beta-r+1\} \\
& 0<x<1, \quad 0<y<1, \sqrt{x(1-y)}+\sqrt{y(1-x)}<1 .
\end{aligned}
$$

A comparison with (2.5) shows that (2.6) holds in a restricted domain and for a particular form of $F_{4}$ but it is interesting to note that (2.6) gives very close bounds and for small values of $x$ and $y$ the lower and upper bounds differ marginally. As an example for the set of values $\alpha=.5, \beta=.7, \gamma=1.2, x=.1, y=.2$, we have from (2.6) $1.111225<$ $F_{4}(\alpha, \beta ; \gamma, \alpha+\beta-\gamma+1 ; x(1-y), y(1-x))=1.114006<1.1167848$. But on the contrary, considering Bailey's result ([3], p. 45(7.1), see also Watson [14])

$$
\begin{aligned}
& \int_{0}^{\infty} t^{\lambda-1} J_{\mu}(a t) J_{\nu}(b t) J_{\rho}(c t) d t \\
= & \frac{2^{\lambda-1} a^{\mu} b^{\nu} \Gamma\{(\lambda+\mu+\nu+\rho) / 2\}}{c^{\lambda+\mu+\nu} \Gamma(\mu+1) \Gamma(\nu+1) \Gamma\left\{1-\frac{1}{2}(\lambda+\mu+\nu-\rho)\right\}} \\
& \times F_{4}\left(\frac{1}{2}(\lambda+\mu+\nu-\rho), \frac{1}{2}(\lambda+\mu+\nu+\rho) ; \mu+1, \nu+1 ; \frac{a^{2}}{c^{2}}, \frac{b^{2}}{c^{2}}\right),
\end{aligned}
$$

provided that

$$
R(\lambda+\mu+\nu+\rho)>0, \quad R(\lambda)<\frac{5}{2}, \quad c>a+b,
$$

one finds on simplification that, though theoretically possible, an inequality for $F_{4}$ does not emerge because of the obvious contradiction in the convergence conditions.

\section{Inequalities for $F_{1}, F_{2}$ and $F_{3}$}

The functions $F_{1}, F_{2}$ and $F_{3}$ are defined by ([6], Vol. I, p. $224(6-8)$ )

$$
F_{1}=F_{1}\left(\alpha, \beta, \beta_{1} ; \gamma ;-x,-y\right)=\sum_{m, n=0}^{\infty} \frac{(\alpha)_{m+n}(\beta)_{m}\left(\beta_{1}\right)_{n}(-x)^{m}(-y)^{n}}{(\gamma)_{m+n} m ! n !}
$$

$\gamma$ is not a negative integer of zero, $|x|<1,|y|<1$,

$$
F_{2}=F_{2}\left(\alpha, \beta, \beta_{1} ; \gamma, \gamma_{1} ;-x,-y\right)=\sum_{m, n=0}^{\infty} \frac{(\alpha)_{m+n}(\beta)_{m}\left(\beta_{1}\right)_{n}(-x)^{m}(-y)^{n}}{(\gamma)_{m}\left(\gamma_{1}\right)_{n} m ! n !}
$$

neither $\gamma$ nor $\gamma_{1}$ is a negative integer or zero, $|x|+|y|<1$, 


$$
\begin{aligned}
F_{3}= & F_{3}\left(\alpha, \alpha_{1}, \beta, \beta_{1} ; \gamma ;-x,-y\right)=\sum_{m, n=0}^{\infty} \frac{(\alpha)_{m}\left(\alpha_{1}\right)_{n}(\beta)_{m}\left(\beta_{1}\right)_{n}(-x)^{m}(-y)^{n}}{(\gamma)_{m+n} m ! n !} \\
& \gamma \text { is not a negative integer or zero, }|x|<1,|y|<1 .
\end{aligned}
$$

Employing (2.3), (3.1)-(3.3) admit respectively the integral representations:

$$
\begin{aligned}
F_{1}= & \frac{1}{\Gamma(\alpha) \Gamma(\beta) \Gamma\left(\beta_{1}\right)} \int_{0}^{\infty} \int_{0}^{\infty} \int_{0}^{\infty} e^{-(r+s+t)} r^{\alpha-1} s^{\beta-1} t \beta_{1}^{-1} \\
& { }_{0} F_{1}\left(-; \gamma ;-(x r s+y r t) d r d s d t, \alpha>0, \beta>0, \beta_{1}>0, \gamma>0, x>0, y>0,(3 .\right. \\
F_{2}= & \frac{1}{\Gamma(\alpha) \Gamma(\beta) \Gamma \beta_{1}} \int_{0}^{\infty} \int_{0}^{\infty} \int_{0}^{\infty} e^{-(r+s+t)} r^{\alpha-1} s^{\beta-1} t_{0}^{\beta_{1}-1} F_{1}(-; \gamma ;-x r s) \\
& { }_{0} F_{1}\left(-; \gamma_{1} ;-y r t\right) d r d s d t, \alpha>0, \beta>0, \beta_{1}>0, \gamma>0, \gamma_{1}>0, x>0, y>0,(3.5)
\end{aligned}
$$

and

$$
\begin{gathered}
F_{3}=\frac{1}{\Gamma(\alpha) \Gamma\left(\alpha_{1}\right) \Gamma(\beta) \Gamma\left(\beta_{1}\right)} \int_{0}^{\infty} \int_{0}^{\infty} \int_{0}^{\infty} \int_{0}^{\infty} e^{-(r+s+t+u)} r^{\alpha-1} s^{\beta-1} t^{\alpha_{1}-1} u^{\beta_{1}-1} \\
\quad \times{ }_{0} F_{1}(-; r ;-(x r s+y t u)) d r d s d t d u \\
\alpha>0, \alpha_{1}>0, \beta>0, \beta_{1}>0, \gamma>0, x>0, y>0 .
\end{gathered}
$$

Therefore, appropriate applications of (1.2) and (2.3) would yield the inequalities:

Theorem 3. If $\alpha>0, \beta>0, \beta_{1}>0, \gamma>0, x>0, y>0$ then

$$
L_{1}<F_{1}<L_{1}+R_{1} / 2
$$

where

$$
\begin{aligned}
& L_{1}=1-\alpha\left(\beta x+\beta_{1} y\right) / \gamma \\
& R_{1}=(\alpha)_{2}\left(x^{2}(\beta)_{2}+y^{2}\left(\beta_{1}\right)_{2}+2 x y \beta \beta_{1}\right) /(\gamma)_{2}
\end{aligned}
$$

Theorem 4. If $\alpha>0, \beta>0, \beta_{1}>0, \gamma>0, \gamma_{1}>0, x>0, y>0$, then

$$
L_{2}<F_{2}<L_{2}+R_{2} / 2
$$

where

$$
\begin{gathered}
L_{2}=1-\alpha\left(\frac{x \beta}{\gamma}+\frac{y \beta_{1}}{\gamma_{1}}\right)+\frac{x y(\alpha)_{2} \beta \beta_{1}}{\gamma \gamma_{1}} \\
R_{2}=\frac{x^{2}(\alpha)_{2}(\beta)_{2}}{(\gamma)_{2}}\left(1-\frac{y(\alpha+2) \beta_{1}}{\gamma_{1}}+\frac{y^{2}(\alpha+2)(\alpha+3)\left(\beta_{1}\right)_{2}}{2\left(\gamma_{1}\right)_{2}}\right)+\frac{y^{2}(\alpha)_{2}(\beta)_{2}}{\left(\gamma_{1}\right)_{2}}\left(1-\frac{x(\alpha+2) \beta}{\gamma}\right),
\end{gathered}
$$

and

Theorem 5. If $\alpha>0, \beta>0, \alpha_{1}>0, \beta_{1}>0, \gamma>0, x>0, y>0$, then

$$
L_{3}<F_{3}<L_{3}+R_{3} / 2
$$


where

$$
\begin{aligned}
& L_{3}=1-\left(x \alpha \beta+y \alpha_{1} \beta_{1}\right) / \gamma \\
& R_{3}=\left(x^{2}(\alpha)_{2}(\beta)_{2}+y^{2}\left(\alpha_{1}\right)_{2}\left(\beta_{1}\right)_{2}+2 x y \alpha \beta \alpha_{1} \beta_{1}\right) /(\gamma)_{2} .
\end{aligned}
$$

It will be remarked here that there are some errors in theorem 6 of Luke [11] and that theorem 6 is obtainable from theorem 4 [11]. Indeed this is so if one writes $\rho=\beta$ and $\rho^{\prime}=\gamma-\beta$ in theorem 4 [11] and the corrected version of Luke's [11] theorem 6 , which is used for comparison, reads as follows:

Theorem 6. If $0<\alpha \leq 1,0<\alpha^{\prime} \leq 1, \gamma>\beta>0, \beta^{\prime}>0, x>0, y>0$, then

$$
\begin{aligned}
& \left(1+\frac{\alpha \beta x}{\gamma}\right)^{-1}\left(1+\frac{\alpha^{\prime} \beta^{\prime} y}{\gamma}\right)^{-1}<F_{3}<\frac{(1-\alpha)(1-c)}{(1+\alpha)}+\frac{(1-\alpha) c\left(c_{1}+c_{2}\right)}{(1+\alpha)} \\
& +\frac{\alpha}{a}(1-c)\left(c_{3}+c_{4}\right)+\frac{\alpha c}{a[x a+f y(1+x a)]}\left(a x\left(c_{3}+c_{4}\right)+f y\left(c_{1}+c_{2}\right)\right),
\end{aligned}
$$

where

$$
\begin{aligned}
a & =\frac{1+\alpha}{2}, \quad c=\frac{2 \alpha^{\prime} \beta^{\prime}(1+\gamma-\beta)}{\left(1+\alpha^{\prime}\right)\left(1+\beta^{\prime}\right)(\gamma+\beta)}, \quad f=\frac{\left(\alpha^{\prime}+1\right)\left(\beta^{\prime}+1\right)}{2(\gamma-\beta+1)}, \\
c_{1} & =\frac{\beta}{\gamma(\gamma-\beta+1)}, \quad c_{2}=\frac{(\gamma-\beta)(1+\gamma)}{\gamma(\gamma-\beta+1)\left(1+v^{\prime}\right)} \\
c_{3} & =\frac{\gamma-\beta}{\gamma(\beta+1)}, \quad c_{4}=\frac{\beta(\gamma+1)}{\gamma /(\beta+1)(1+v)}, \\
v & =\frac{(1+\alpha)(1+\beta) x}{2(\gamma+1)}, \quad v^{\prime}=\frac{\left(1+\alpha^{\prime}\right)\left(1+\beta^{\prime}\right) y}{2(\gamma+1)} .
\end{aligned}
$$

\section{Numerical verification}

Table II below provides numerical verification of theorem 3, 4, 5 and 6 and also compares our results with those of Luke [11]. For instance, for the set of values as given by table I:

Table $\mathbb{I}$

\begin{tabular}{cccccccccc}
\hline & S.No. & $\alpha$ & $\alpha_{1}$ & $\beta$ & $\beta_{1}$ & $r$ & $r_{1}$ & $x$ & $y$ \\
\hline \multirow{2}{*}{$F_{1}$} & $(1)$ & 0.6 & - & .4 & .35 & 2.0 & - & .2 & .3 \\
& & & & & & & & & \\
& $(2)$ & 0.5 & - & .4 & .3 & 0.45 & - & .05 & .01 \\
\hline \multirow{2}{*}{$F_{2}$} & $(3)$ & 0.6 & - & .4 & .35 & 2.0 & 2.5 & .2 & .3 \\
& $(4)$ & 1.1 & - & .5 & .3 & .4 & .25 & .1 & .05 \\
\hline \multirow{2}{*}{$F_{3}$} & $(5)$ & 0.6 & 0.5 & .4 & .35 & 2.0 & - & .2 & .3 \\
& $(6)$ & 1.1 & 1.3 & .7 & .3 & .6 & - & .05 & .01 \\
\hline
\end{tabular}




\section{Table II}

\begin{tabular}{ccc}
\hline S. No. & Our inequality Luke's inequality & \\
\hline 1. & $.9445<F_{1}=.95018<.95103$ & $.9474<F_{1}=.95018<1.00076$ \\
& & $([11)$,eq. $(4))$ \\
2. & $.9744<F_{1}=.975306<.97534$ & - \\
3. & $.95241<F_{2}=.955751<.9561$ & $.9531<F_{2}=.955751<.9589$ \\
& & $([11])$, eq. $(12))$ \\
4. & $.813825<F_{2}=.82763<.8434225$ & -- \\
5. & $.94975<F_{3}=.954072<.96461$ & $.95158<F_{3}=.954072<.961532$ \\
& & (corrected version of \\
& & Luke' theorem $6 ;$ see \\
& & our equation 3.10$)$ \\
6. & $.92935<F_{3}=.932941<.936928$ & -
\end{tabular}

The numerical computation given above not only points out the sharpness of our results in some cases but also verifies these inequalities in the extended domain not conceivable by Luke's inequalities.

\section{Some more inequalities}

(3.4) and (3.5) can also be written in the form

$$
F_{1}=\frac{1}{\Gamma(\beta) \Gamma\left(\beta_{1}\right)} \int_{0}^{\infty} \int_{0}^{\infty} e^{-(r+s)} r^{\beta-1} s^{\beta_{1}-1}{ }_{1} F_{1}(\alpha ; \gamma ;-(x r+y s)) d r d s
$$

and

$$
F_{2}=\frac{1}{\Gamma(\alpha)} \int_{0}^{\infty} e^{-r} r_{1}^{\alpha-1} F_{1}(\beta ; \gamma ;-x r){ }_{1} F_{1}\left(\beta_{1} ; \gamma_{1} ;-y r\right) d r
$$

under the same set of conditions. Now approximating ${ }_{1} F_{1}$ 's by Luke's ([10], 5.5) results we have respectively the theorems:

Theorem 7. Let $\gamma>\alpha>0, \beta>0, \beta_{1}>0, x>0, y>0$, then

$$
\begin{aligned}
\left(1+\frac{\alpha}{\gamma} x\right)^{-\beta}\left(1+\frac{\alpha}{\gamma} y\right)^{-\beta_{1}} & <F_{1}\left(\alpha, \beta, \beta_{1} ; \gamma ;-x-y\right) \\
& <\left(1-\frac{\alpha}{\gamma}\right)+\frac{\alpha}{\gamma}(1+x)^{-\beta}(1+y)^{-\beta_{1}},
\end{aligned}
$$

Theorem 8. Let $\gamma>\alpha>0, \beta>0, \beta_{1}>0, x>0, y>0$ then

$$
\begin{aligned}
\left(1+\frac{\beta}{\gamma} x+\frac{\beta_{1}}{\gamma} y\right)^{-\alpha} & <F_{2} \\
& <\left(1-\frac{\beta}{\gamma}\right)\left(1-\frac{\beta_{1}}{\gamma_{1}}\right)+\frac{\beta}{\gamma}\left(1-\frac{\beta_{1}}{\gamma_{1}}\right)(1+x)^{-\alpha} \\
& +\left(1-\frac{\beta}{\gamma}\right) \frac{\beta_{1}}{\gamma_{1}}(1+y)^{-\alpha}+\frac{\beta \beta_{1}}{\gamma \gamma_{1}}(1+x+y)^{-\alpha}
\end{aligned}
$$


Numerically for the set of values appended in the table $I$ it is seen that in the common domain of validity these results provide improved lower bound even than those of Luke [11]. Similarly inequalities for positive real arguments are given as:

Theorem 9. Let $\gamma>\alpha>0, \beta, \beta_{1}>0,0<x<1,0<y<1$, then

$$
\begin{aligned}
\left(1-\frac{\alpha}{\gamma} x\right)^{-\beta}\left(1-\frac{\alpha}{r} y\right)^{-\beta_{1}} & <F_{1}\left(\alpha, \beta, \beta_{1} ; \gamma ; x, y\right) \\
& <\left(1-\frac{\alpha}{\gamma}\right)+\frac{\alpha}{\gamma}(1-x)^{-\beta}(1-y)^{-\beta_{1}} .
\end{aligned}
$$

Theorem 10. Let $\gamma>\alpha>0, \beta, \beta_{1}<0,0<x<1,0<y<1$, then

$$
\begin{aligned}
& \left(1-\frac{\beta}{\gamma} x-\frac{\beta_{1}}{\gamma} y\right)^{-\alpha}<F_{2}\left(\alpha, \beta, \beta_{1} ; \gamma, \gamma_{1} ; x, y\right)<\left(1-\frac{\beta}{\gamma}\right)\left(1-\frac{\beta_{1}}{\gamma_{1}}\right) \\
& +\frac{\beta}{\gamma}\left(1-\frac{\beta_{1}}{\gamma}\right)(1-x)^{-\alpha}+\left(1-\frac{\beta}{\gamma}\right) \frac{\beta_{1}}{\gamma}(1-y)^{-\alpha}+\frac{\beta \beta_{1}}{\gamma \gamma}(1-x-y)^{-\alpha} .
\end{aligned}
$$

and

Theorem 11. Let $\alpha, \beta, \beta_{1}, \gamma>0,0<x<1,0<y<1$, then

$$
1+\left(\frac{x \alpha \beta+y \alpha_{1} \beta_{1}}{\gamma}\right)<F_{3}\left(\alpha, \alpha_{1}, \beta, \beta_{1} ; x, y\right)<1+2\left(\frac{x \alpha \beta+y \alpha_{1} \beta_{1}}{\gamma}\right) .
$$

Theorem 9 and 10 can also be obtained in light of (3.4) and (3.5), however, the details are omitted for the reasons of brevity. Here it is observed that the advantage of these inequalities is that, in the first place they are precise in contrast to the unwieldy results available earlier and secondly hold under weaker conditions and give sharper bounds in the common domain of validity.

\section{Acknowledgement}

First author expresses his thank to UGC for financial assistance.

\section{References}

[1] W. N. Bailey, "A reducible case of the fourth type of Appell's hypergeometric functions of two variables," Quart. J. Math. (Oxford), 4 (1933), 305-308.

[2] W. N Bailey, Generalized Hypergeometric Series, Cambridge University Press 1935.

[3] W. N. Bailey, "Some infinite integrals involving Bessel functions," Proc. London Math. Soc. (Oxford), 40 (1936), 37-48.

[4] W. N. Bailey, "On the double integral representation of Appell's function $F_{4}$," Quart. J. Math. (Oxford), 12 (1941), 12-14.

[5] J. L. Burchnall and T. W. Chaundy, "Expansions of Appell's double hypergeometric functions", Quart. J. Math. (Oxford), 11 (1940), 249-270. 
[6] A. Erdélyi et al., Higher Transcendental Functions, Vols. I, II, McGraw-Hill, New York 1953.

[7] C. M. Josh and J. P. Rya, "Some inequalities for the Appeal's hypergeometric functions $F_{1}, F_{2} "$, Jnanabha 9/10 (1980), 179-186.

[8] C. M. Joshi and S. K. Bissu, "On the inequalities of Appell functions," Boll. U. M. I., 7 (7-B) (1993), 71-85.

[9] Y. L. Luke, The Special Functions and Their Approximations, Vol. II, Academic Press, New York, 1969.

[10] Y. L. Luke, "Inequalities for generalized hypergeometric functions," J. Approx. Theory, $5(1972), 41-65$.

[11] Y. L. Luke, "Inequalities for generalized hypergeometric functions of two variables," $J$. Approx. Theory, 11 (1974), 73-84.

[12] H. M. Srivastava and Per W. Karlsson, Multiple Gaussian Hypergeometric Series, Ellis Horwood, Chichester, 1985.

[13] G. N. Watson, "The product of two hypergeometric functions," Proc. London Math. Soc., 20 (1992), 189-195.

[14] G. N. Watson, A Treatise on the Theory of Bessel Functions, Cambridge University Press, 1944.

Department of Mathematics, Sukhadia University, Udaipur, India. 